\title{
Stent deployment protocol for optimized real-time visualization during endovascular neurosurgery
}

\author{
Michael A. Silva, BA, Alfred P. See, MD, Hormuzdiyar H. Dasenbrock, MD, Ramsey Ashour, MD, \\ Priyank Khandelwal, MBBS, Nirav J. Patel, MD, Kai U. Frerichs, MD, and \\ Mohammad A. Aziz-Sultan, MD
}

Department of Neurosurgery, Brigham and Women's Hospital, Harvard Medical School, Boston, Massachusetts

\begin{abstract}
Successful application of endovascular neurosurgery depends on high-quality imaging to define the pathology and the devices as they are being deployed. This is especially challenging in the treatment of complex cases, particularly in proximity to the skull base or in patients who have undergone prior endovascular treatment. The authors sought to optimize real-time image guidance using a simple algorithm that can be applied to any existing fluoroscopy system. Exposure management (exposure level, pulse management) and image post-processing parameters (edge enhancement) were modified from traditional fluoroscopy to improve visualization of device position and material density during deployment. Examples include the deployment of coils in small aneurysms, coils in giant aneurysms, the Pipeline embolization device (PED), the Woven EndoBridge (WEB) device, and carotid artery stents. The authors report on the development of the protocol and their experience using representative cases.
\end{abstract}

The stent deployment protocol is an image capture and post-processing algorithm that can be applied to existing fluoroscopy systems to improve real-time visualization of device deployment without hardware modifications. Improved image guidance facilitates aneurysm coil packing and proper positioning and deployment of carotid artery stents, flow diverters, and the WEB device, especially in the context of complex anatomy and an obscured field of view.

https://thejns.org/doi/abs/10.3171/2016.4.JNS16194

KEY WORDS aneurysm coiling; angiography; carotid stent; flow diversion; fluoroscopy; vascular disorders

$\mathrm{P}$ RECISE image guidance is required for safe and definitive endovascular treatment, including aneurysm treatment with coils, flow diversion, or intrasaccular devices (Woven EndoBridge [WEB]), and carotid artery stent placement. ${ }^{3,5,6,11}$ As endovascular approaches are applied to more complex cases, they have increasingly relied on nuanced devices that require precise sizing, positioning, and wall apposition to function optimally.

Among other challenges, endovascular approaches can be limited by suboptimal visualization during device deployment, resulting in complications and recurrent pathology. Proper device positioning, optimal wall apposition, and appreciation for coil packing density necessitate high-resolution, real-time image guidance. ' For example, as many as $28 \%$ of flow-diverting stents may require repositioning due to incomplete wall apposition due to suboptimal initial device deployment. ${ }^{10}$ Optimized image guidance has been shown to improve WEB device visualization and facilitate deployment and positioning. ${ }^{1,14}$ Device deployment is further complicated when previously deployed endovascular devices or skull base anatomy obstruct the field of view.

Other specialties have recognized the limitations of traditional fluoroscopy for endovascular device deployment. Interventional cardiologists have used digital manipulation of standard x-ray images to optimize real-time image guidance during device deployment, leading to improved stent placement, expansion, and wall apposition in percutaneous coronary intervention. ${ }^{17}$ Imaging protocols can be manipulated to improve visualization during endovascular device deployment to address the limitations of relying on pre-deployment imaging and on suboptimal traditional fluoroscopy in real time.

Improvements in neuroendovascular imaging have primarily described hardware modifications such as im- 
provements in detectors and software upgrades inherited from other specialties, which minimize radiation but do not necessarily improve the quality of visualization. We worked directly with the engineering and deployment team at Siemens Healthcare to optimize the image capture exposure parameters and image post-processing to improve real-time visualization of device deployment for use in cerebrovascular applications. These techniques are not included on standard installation of neuroangiographic systems but are based on core principles of cerebrovascular angiography and can be applied to many generations of angiography systems from various companies to improve device visualization during critical portions of neuroendovascular interventions. Here, we present a series of demonstrative cases in which this novel imaging protocol developed for neuroendovascular specialists improved device visualization and intraprocedural clinical decision making for aneurysm coiling, Pipeline embolization device (PED) deployment, WEB device deployment, and carotid artery stent deployment.

\section{Methods}

After expert optimizing of fluoroscopic settings by the manufacturer, we further requested a customized protocol. Over multiple sessions with the system deployment team, we developed a custom protocol to visualize the devices in neuroangiography within the cranial space. Of note, this protocol sacrificed quality of contrast visualization for the purpose of device visualization. We serially adapted the 2 modifiable parameters in the fluoroscopy system to create the stent deployment protocol: exposure management, and image post-processing. In comparison with a typical angiographic run and the fluoroscopic run, the stent deployment protocol used a voltage of $70 \mathrm{kV}$ (compared with $73 \mathrm{kV}$ for angiographic and $73 \mathrm{kV}$ for fluoroscopic protocols, respectively), a pulse width of $25 \mathrm{msec}$ ( $40 \mathrm{msec}, 16$ $\mathrm{msec}$ ), a dose of $0.54 \mathrm{microGy} / \mathrm{frame}$ (2.4 microGy/frame, $55 \mathrm{nGy} /$ pulse). The image post-processing was set to edge enhancement $90 \%(30 \%, 25 \%)$, window center 1600 and width 1900 (1900 and 2700, 1650 and 2475), with linear windowing (sigmoid, linear), gamma correction G05/C1 (gamma log, G06/C3), gain correction $-1.0 \mathrm{EP}$ (0.0 EP, $0.0 \mathrm{EP}$ ), K-factor auto7 (off, auto6). Since the imaging was performed without a subtraction masking, only native processing was used.

The most critical parameters in comparison with angiography include the pulse width, which decreases the blurring of fine details from temporal summation; edge enhancement, which increases the visual contrast between the device material and the surrounding blank space; windowing, which sacrifices low-density super-saturation for further improvement of contrast within the range of device density; and gamma correction, which further maximizes the signal gradient in the range relevant to the device density.

In comparison with a fluoroscopic run, the pulse width was slightly increased, improving the detector sensitivity, with increased edge enhancement for better device-blank space visual contrast, narrow window width to sacrifice super-saturation for improved contrast within the device density range, and gamma correction with maximum signal gradient at the range relative to the device density.

These settings were also applied to a GE Healthcare system that is used for neuroangiography and neurointervention.

We used this protocol in addition to our standard fluoroscopy protocol during placement of the penultimate and ultimate coils in an aneurysm coiling procedure and during intermittent critical stages of carotid artery stent, WEB, and PED deployments. A representative example of this application in each device was reviewed in a retrospective manner. We reviewed intraoperative images and selected magnified views of the devices for quantitative comparison. We quantified the contrast ratio within representative regions of the devices or coils as visualized using manufacturer-optimized fluoroscopy versus the stent deployment protocol using the "Colour Contrast Analyser" from the Paciello Group and the National Institute on Disability, Independent Living, and Rehabilitation Research.

\section{Illustrative Cases}

\section{Case 1: Coil Deployment in a Small Aneurysm}

A patient in the 5th decade of life with a history of hypertension and depression presented with acute-onset severe headache and diffuse cisternal subarachnoid hemorrhage. Computed tomography angiography demonstrated a $3 \times 2-\mathrm{mm}$ narrow-necked inferoposteriorly projecting anterior communicating artery aneurysm which was confirmed on angiogram (Fig. 1A). A Target 360 Ultra 2.5$\mathrm{mm} \times 4-\mathrm{cm}$ coil and a Target 360 Nano $1-\mathrm{mm} \times 2-\mathrm{cm}$ coil (Stryker Neurovascular) were deployed into the aneurysm using standard fluoroscopic guidance (Fig. 1B) with interval confirmation using the stent deployment protocol. At the final coil deployment, the density of coil packing was reassessed with the stent deployment protocol (Fig. 1C). We observed a significantly higher contrast ratio of 11.22 using stent deployment compared with a contrast ratio of 1.67 with fluoroscopy $(\mathrm{p}<0.001)$.

Real-time visualization of individual loops of the coil allowed confident expeditious deployment of the coil within the aneurysm and appreciation for the tension and position of each coil loop and the catheter tip. Improved visualization of the individual loops also provides greater appreciation for packing density to minimize risk of recanalization. ${ }^{16}$

\section{Case 2: Carotid Artery Stent Deployment}

A patient in the 7th decade of life with a history of coronary artery disease presented with left hemispheric transient ischemic attacks and unstable angina and 80\%-90\% severe focal stenosis within the proximal left internal carotid artery (ICA). Cardiac catheterization demonstrated a tandem $40 \%$ and $80 \%$ stenosis of the right circumflex artery, which was the attributed source of the unstable angina. Given the patient's high-risk comorbidities, the decision was made to pursue carotid artery revascularization by stent placement.

Biplane digital subtraction angiography was completed, demonstrating the carotid artery stenosis (Fig. 2A). A distal protection device was deployed prior to pre-stent 

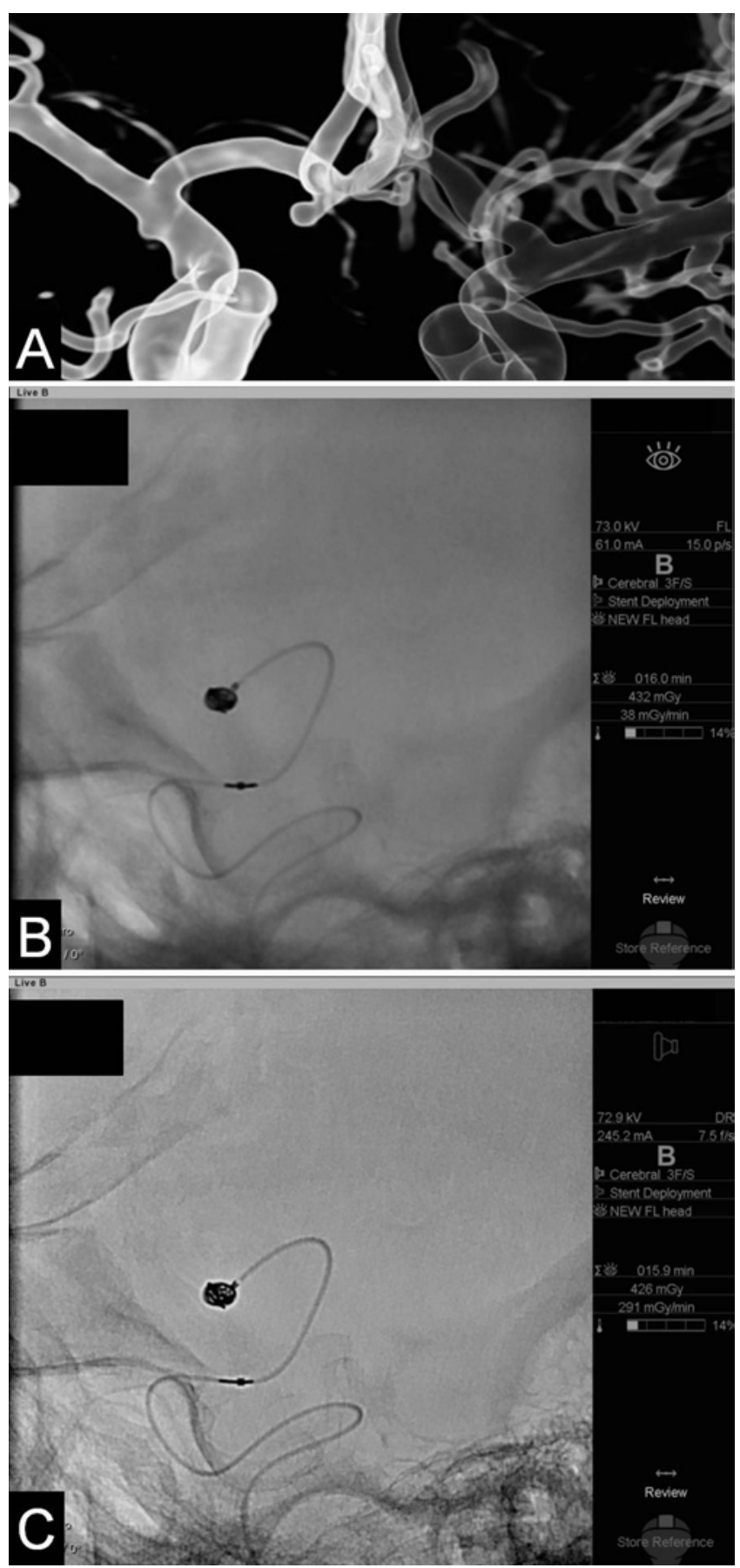

FIG. 1. Illustrative Case 1. A: Fused 3D reconstruction of right and left ICA rotational angiograms demonstrating the anterior communicating artery aneurysm projecting inferolaterally. B: Standard fluoroscopic visualization of the coil mass. C: Stent deployment visualization of the coil mass demonstrating the improved assessment of packing density. The stent deployment sequence, from which this figure is derived, lasted 2 seconds and accumulated $7.7 \mathrm{mGy}$ of radiation. A digital subtraction acquisition of the same projection and magnification lasting 5 seconds accumulated $36.7 \mathrm{mGy}$. During this procedure, there were 26.8 minutes of fluoroscopic acquisition, accumulating $2307 \mathrm{mGy}$ of radiation.
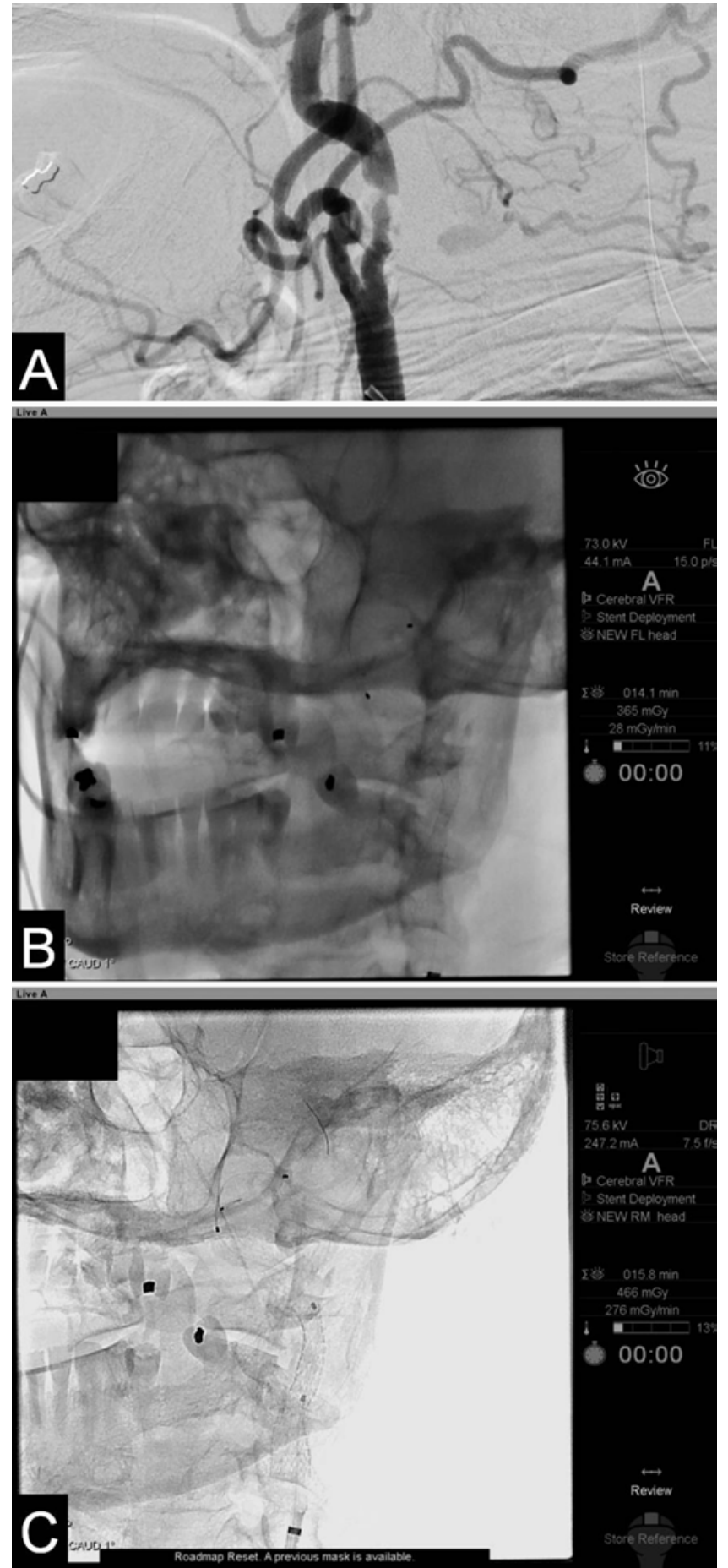

FIG. 2. Illustrative Case 2. A: Lateral projection of left common carotid artery (CCA) angiogram demonstrating left ICA stenosis. B: Standard fluoroscopic visualization of the deployed carotid artery stent. C: Stent deployment visualization of the deployed carotid artery stent demonstrating improved visualization, particularly of the proximal and distal termini of the stent tines. The stent deployment sequence, from which this figure is derived, lasted 2 seconds and accumulated $9.2 \mathrm{mGy}$ of radiation. A comparable digital subtraction acquisition of the same projection and magnification lasting 4 seconds accumulated $20.8 \mathrm{mGy}$. During this procedure, there were 30.5 minutes of fluoroscopic acquisition, accumulating $825.1 \mathrm{mGy}$ of radiation. 


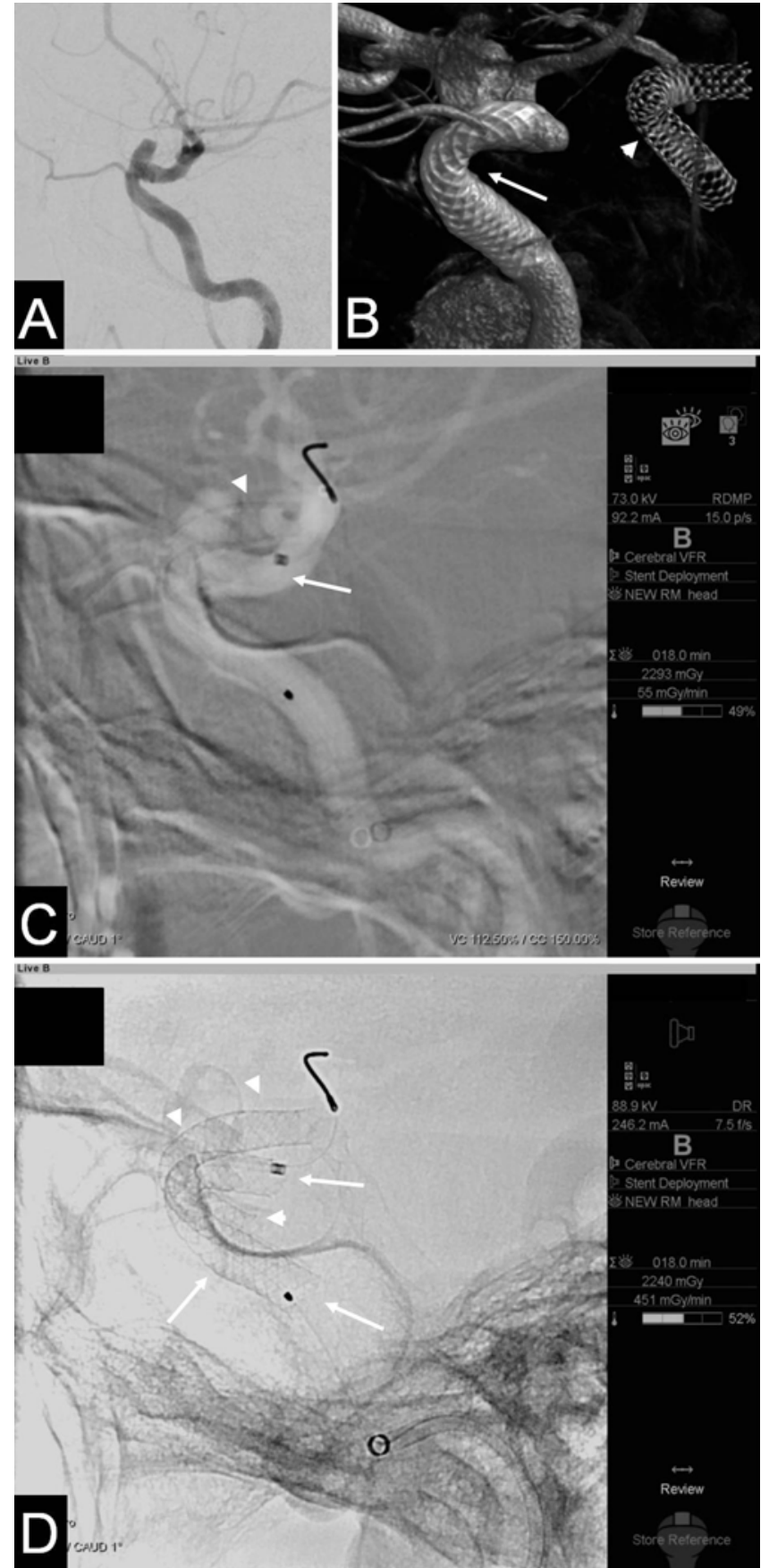

FIG. 3. Illustrative Case 3. A: Lateral right CCA angiogram demonstrating the right ophthalmic artery aneurysm. B: $3 D$ reconstruction of right ICA angiogram with dual-volume protocol demonstrating bilateral pipeline stents (arrow indicates ipsilateral stent; arrowhead indicates contralateral stent). C: Standard fluoroscopic visualization of the deployed right ICA pipeline (arrow indicates ipsilateral stent, arrowhead indicates contralateral stent). D: Stent deployment (SD) visualization of the deployed right ICA stent demonstrating improved visualization of the ends of the stent (arrows indicate ipsilateral stent, arrowheads indicate contralateral stent), cell density along each segment of the stent, and disparate torsional forces which may lead to stent twisting or kinking. The SD sequence, from which this figure is derived, lasted 18 seconds and accumulated $136 \mathrm{mGy}$ of radiation. A comparable digital subtraction acquisition of the same projection and magnification lasting 7 seconds accumulated $51.5 \mathrm{mGy}$. During this procedure, there were 30.6 minutes of fluoroscopic acquisition, accumulating $4857 \mathrm{mGy}$ of radiation. balloon angioplasty. A $7 \times 30-\mathrm{mm}$ ev3 Protégé stent was advanced across the stenosis. Under a combination of standard fluoroscopic and stent deployment visualization, the stent was unsheathed (Fig. 2B and C). We observed a significantly higher contrast ratio of 2.27 using stent deployment compared with a contrast ratio of 1.28 with fluoroscopy ( $\mathrm{p}<0.001)$.

With serial evolution of carotid artery stents, device visualization has become less of a challenge. In comparison with the standard angiographic acquisition, the stent deployment protocol did not provide significant improvement in visualization of the stent. However, in comparison with the standard fluoroscopic loop, there was significant improvement in the visualization of the ends of the stent where the device material is less dense.

\section{Case 3: Flow-Diverter Deployment}

A patient in the 7th decade of life with a significant medical history, most notable for atrial fibrillation, congestive heart failure, Type 2 diabetes, thoracic aortic aneurysm, and hypertension, presented with transient visual loss and slurred speech lasting 30 minutes. During workup for stroke, the patient underwent MRI of the brain with and without contrast, which demonstrated left cervical ICA atherosclerosis with 50\% stenosis, and multiple intracranial aneurysms: a 6-mm right ophthalmic artery aneurysm, a 9-mm left ophthalmic artery aneurysm, a 3 -mm left superior hypophyseal artery aneurysm, and a 3-mm basilar bifurcation aneurysm. Despite the atypical history, due to the size of the aneurysm, the decision was made to perform aneurysm repair, first of the left ophthalmic artery aneurysm, then of the right ophthalmic artery aneurysm (Fig. 3A), with bilateral flow diverters.

A $3.5 \times 16-\mathrm{mm}$ PED was deployed in the left ophthalmic segment. The patient returned 5 weeks later for flow diversion of the right ophthalmic artery aneurysm using a $4 \times 16-\mathrm{mm}$ PED beginning in the right $\mathrm{M}_{1}$ segment and withdrawn into the right ophthalmic segment. During further stent unsheathing within the ophthalmic segment, the stent deployment protocol was used to visualize the distal end of the stent as well as the individual tines to ensure good wall apposition around the turns of the right ICA (Fig. 3D). A post-deployment biplane DS angiogram and rotational angiogram with subtraction masking were obtained (Fig. 3B). A comparison of the standard fluoroscopic run (Fig. 3C) to the stent deployment protocol (Fig. 3D) demonstrates significantly improved visualization of the newly deployed stent distinct from the prior stent and from the skull base anatomy. We observed a significantly higher contrast ratio of 1.59 using the stent deployment protocol compared with a contrast ratio of 1.05 with fluoroscopy ( $\mathrm{p}<0.001)$.

PEDs are compliant, which enables them to conform to the cerebrovascular tortuosity; thus, high-quality image guidance is crucial for attaining proper positioning upon deployment. During deployment, torsional forces can twist and kink the stent while longitudinal forces can modulate the mesh density. Real-time visualization of the catheterstent interface permits modulation of these forces to deliver the stent successfully. Concurrently, continuous accurate visualization of the distal end of the stent ensures adequate 


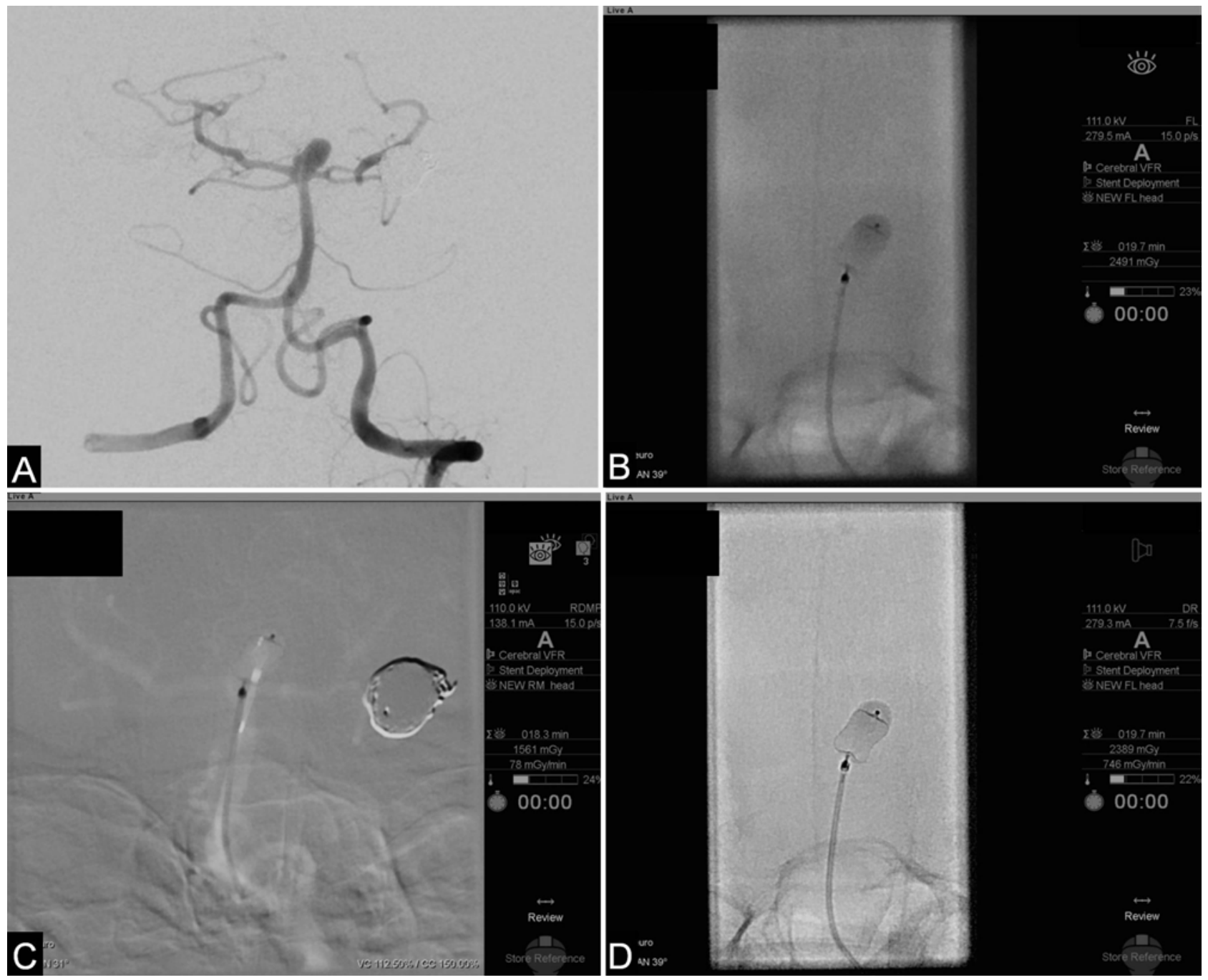

FIG. 4. Illustrative Case 4. A: Left vertebral artery angiogram demonstrating the basilar bifurcation aneurysm. B: Fluoroscopic visualization of the WEB device before detachment. C: Roadmap visualization of the WEB device before detachment. D: Stent deployment (SD) visualization of the WEB device before detachment demonstrating improved visualization of the device architecture and particularly of the shoulders of the device at the neck of the aneurysm. The SD sequence, from which this figure is derived, lasted 13 seconds and accumulated $176 \mathrm{mGy}$ of radiation. A comparable digital subtraction acquisition of the same projection and magnification lasting 5 seconds accumulated $56 \mathrm{mGy}$. During this procedure, there were 38.7 minutes of fluoroscopic acquisition, accumulating $4890 \mathrm{mGy}$ of radiation.

support and aneurysm neck coverage. Furthermore, postdeployment repositioning requires an appreciation of stent wall irregularities to suggest poor wall apposition. Realtime high-definition imaging of the distal stent and stent walls enable expedited and accurate stent deployment.

\section{Case 4: WEB Device Deployment}

A patient in the 5th decade of life without significant medical history who was taking aspirin for cardioprotection presented with acute-onset headache in the evening and was found having collapsed at home. During workup, a noncontrast CT scan of the head demonstrated aneurysmal subarachnoid hemorrhage and left temporal intracerebral hematoma, and the examination findings corresponded to Hunt and Hess Grade IV. A follow-up CT angiogram demonstrated an $11.6 \times 8.6 \times 7.7-\mathrm{mm}$ left posterior com- municating artery aneurysm, which was coiled without residual as the source of her subarachnoid hemorrhage. At that time the patient was also found to have an unruptured basilar tip aneurysm (Fig. 4A). The patient recovered very well with a modified Rankin Scale score of 2: she had some impulsiveness, a slight right facial droop, and subtle right grip weakness. Following recovery from the subarachnoid hemorrhage and associated angiographic vasospasm, the patient was evaluated for WEB endosaccular treatment of the basilar tip aneurysm within a research protocol.

Under standard fluoroscopic and roadmap guidance a $6 \times 4-\mathrm{mm}$ WEB SL device was deployed within the basilar bifurcation aneurysm (Fig. 4B and C). During catheter advancement repositioning of the WEB device, the stent deployment protocol was used for improved visualization of the shoulders of the device and the origins of the pos- 
terior cerebral arteries bilaterally (Fig. 4D). We observed a significantly higher contrast ratio of 1.67 using stent deployment compared with a contrast ratio of 1.10 with fluoroscopy ( $<$ < 0.001).

Despite multiple iterations of device refinement, device visualization is based on the radiopaque platinum markers at the tip, the base, and in certain models, within the core of the WEB device. Although formed from nitinol wire, the mesh walls of the stent have limited visibility during fluoroscopic runs, which are used during the deployment. The stent deployment protocol enabled better visualization of wall apposition, WEB shoulder irregularity, and protrusion of the device at the aneurysm neck during the entirety of the deployment.

\section{Discussion}

New devices used in endovascular neurosurgery have increased demand for high-quality, real-time image guidance to accommodate the nuanced structures and approaches that these new devices entail. Visualization of device deployment is a key barrier to obtaining the precise sizing, placement, and wall apposition that are necessary for these devices to function properly. As many as $37 \%$ of aneurysms treated with the WEB device exhibit recanalization. ${ }^{4,9}$ Similarly, up to $28 \%$ of flow-diverting stents require repositioning due to incomplete wall apposition. ${ }^{10}$ These failure rates are potentially partly a consequence of suboptimal visualization.

The stent deployment protocol optimizes the exposure parameters and image post-processing to attain superior intraprocedural visualization. No comparable image optimization protocols are currently available as standard configuration and optimization through fluoroscopy manufacturers. Our experience with the stent deployment protocol suggests that these optimized parameters can improve image guidance and device deployment in technically challenging cases, including coil embolization of small aneurysms and deployment of intracranial flow-diverting stents, WEB devices, and carotid artery stents. The stent deployment protocol can be applied to any existing system. We have used this protocol during nearly all flow diverter and WEB device deployments over a 6-month period, as well as during approximately 5\% of aneurysm coiling procedures. We observed an increased contrast ratio in intraoperative images captured when using the stent deployment protocol versus fluoroscopy, allowing for better appreciation of coil density and device features during deployment.

The parameters for this protocol were refined via an interactive adjustment process involving the senior author (M.A.A.S.) and the technician from Siemens responsible for device installation and troubleshooting. Our group also discussed these results with technicians from GE Healthcare, who then adapted these settings of edge enhancement and windowing, while maintaining their optimized exposure management protocol. Application of this protocol would require physician collaboration with the technical staff from the vendor team, whether from GE Healthcare, Philips Healthcare, Siemens Healthcare, or Toshiba Corporation.

Very small aneurysms can be difficult to pack densely with coils, requiring visualization of the position of each coil being placed. ${ }^{2,12}$ The stent deployment protocol allows for better visualization of the remaining endosaccular space and the precise conformation of coil breaking upon deployment. Similarly, coiling very large aneurysms can be challenging because the presence of many overlapping coils can obscure the actual density of coils and the degree of aneurysm occlusion. Our protocol might also improve coiling of large aneurysms by optimizing visualization of the coils within the aneurysm sac.

The utility of a carotid stent relies on proper placement within the vessel. While carotid stenting can be more forgiving than intracranial stenting due to larger vessel diameter and less complex vessel geometry, the irretrievable nature of these stents means it is imperative that the device be placed properly. Given the large vessel caliber, our protocol might not be particularly helpful for improving wall apposition. However, it can improve the visualization of the distal and proximal ends of the stent to ensure that it provides sufficient coverage over the area of stenosis.

The WEB device is typically well visualized by traditional imaging, and it is retrievable, allowing for formal angiography runs to assess proper placement. Relative to ophthalmic segment aneurysms, MCA bifurcation aneurysms and high basilar bifurcation aneurysms are farther from the skull base. This distance improves the quality of the fluoroscopy for device visualization. The weave of the WEB is highly visible and accentuated by titanium markers at the tip and the base of the device. The lowest visibility occurs at the en-face surface of the WEB when deployed, and intermediate visibility occurs at the lateral borders and at the shoulder of the deployed device where several layers of the mesh overlap. In wide-necked aneurysms for which it was specifically designed, protrusion of the device into the parent vessel can risk vessel occlusion, thrombosis, and stroke. The stent deployment protocol can be used to improve visualization of WEB positioning at the aneurysm shoulder, where the mesh density and therefore fluoroscopic opacification is intermediate.

Flow diverters present perhaps the most useful application of the stent deployment protocol. Since flow diverters are often used for aneurysms located near the skull base (e.g. ophthalmic aneurysms, cavernous carotid aneurysms), traditional fluoroscopy imaging can be obscured by the nearby bone. Furthermore, multiple flow diverters may be placed for complex reconstructions or for recurrences, and the presence of overlying stents can further obscure the device position during standard fluoroscopy. In one case, we found that our modified protocol greatly facilitated visualization of a PED deployment despite the presence of a second contralateral stent in the field of view. Often, oversizing of PEDs or balloon angioplasty is used to combat poor wall apposition, but this approach carries the risk of decreased flow diversion due to increased wall porosity at submaximal stent opening. ${ }^{8}$ Improved imaging has the potential to improve sizing and diminish the need to oversize the stents. Our experience using the stent deployment protocol suggests that it can be helpful for visualization of the distal and proximal ends of flow diverters during deployment and, albeit to a lesser degree, for assessing wall apposition. 


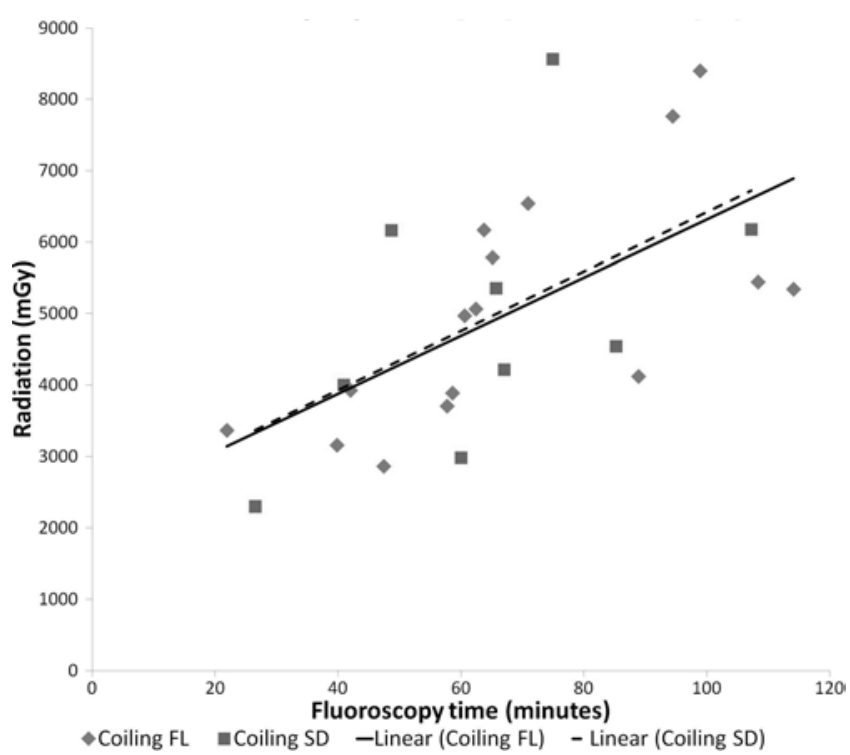

FIG. 5. Plot of radiation as a function of fluoroscopic duration during aneurysm coiling from a 12-month period spanning the application of the stent deployment (SD) protocol. FL = fluoroscopy.

The stent deployment protocol improves device visualization but involves an approximately sevenfold increased radiation exposure rate to the patient compared with traditional fluoroscopy. Therefore, it should be used in moderation and only when improved image quality is most necessary, such as during final device deployment. In our experience, we have not found significantly increased overall case radiation dose when using the stent deployment protocol. The magnification and duration of fluoroscopy are the most significant factors in overall radiation dose of coiling cases, and there is no change in the linear relationship between radiation and imaging duration as demonstrated by linear regression (Fig. 5). In our practice, there is an overall substitution of the acquisition angiography protocol for the stent deployment protocol. The cumulative imaging time is not statistically significantly different (mean 68 minutes without the stent deployment protocol versus 64 minutes with it). There was only one Pipeline embolization device placed without using the stent deployment protocol, and there were no WEB devices placed without using it.

Although not illustrated, the principles of improved image guidance demonstrated by the stent deployment protocol would be reasonably inferred to carry over to other devices under development and under trial at this time, such as PulseRider or Barrel vascular reconstruction device, or to stent retrievers used in mechanical thrombectomy. ${ }^{13,15}$

\section{Conclusions}

Based on adjustment to the acquisition technique as well as modifications to the image processing, the stent deployment protocol can be applied at centers with systems from different generations and from different manufacturers. This protocol has been specifically adjusted to optimize visualization of the cerebrovascular anatomy. While many clinicians are engaged in trials of advancing device tech- nology, imaging quality is an area ripe with potential for increased neurointerventionalist involvement. Although fluoroscopy systems are optimized during setup and configuration, manufacturers are not currently able to further optimize for these clinical applications without close collaboration and customization with the interventionalist. During the experience of the senior author (M.A.A.S.) in proctoring PED usage, visualization has been a universal challenge despite high-quality and well-maintained fluoroscopic systems. Stent deployment, a simple imagecapture and processing algorithm that can be applied to any fluoroscopy system, improves image guidance for aneurysm coiling, endosaccular device deployment, flowdiverter deployment, and carotid artery stent deployment. The protocol is of the greatest utility for visualizing flow diverters and coil embolization of small aneurysms, especially near the skull base.

\section{Acknowledgments}

Henry A. Muler, MSEE, of Siemens Medical Solutions, USA, and Peter O'Rourke, Lead Neurointerventional Technician at Brigham and Women's Hospital, collaborated extensively with the group in optimizing the protocol prior to clinical use and provided education to the team about principles of fluoroscopy.

\section{References}

1. Caroff J, Mihalea C, Neki H, Ruijters D, Ikka L, Benachour $\mathrm{N}$, et al: Role of C-arm VasoCT in the use of endovascular WEB flow disruption in intracranial aneurysm treatment. AJNR Am J Neuroradiol 35:1353-1357, 2014

2. Cho YD, Jeon JP, Rhim JK, Park JJ, Yoo RE, Kang HS, et al: Progressive thrombosis of small saccular aneurysms filled with contrast immediately after coil embolization: analysis of related factors and long-term follow-up. Neuroradiology 57:615-623, 2015

3. Diethrich EB, Gordon MH, Lopez-Galarza LA, RodriguezLopez JA, Casses F: Intraluminal Palmaz stent implantation for treatment of recurrent carotid artery occlusive disease: a plan for the future. J Interv Cardiol 8:213-218, 1995

4. Fiorella D, Arthur A, Byrne J, Pierot L, Molyneux A, Duckwiler G, et al: Interobserver variability in the assessment of aneurysm occlusion with the WEB aneurysm embolization system. J Neurointerv Surg 7:591-595, 2015

5. Fiorella D, Lylyk P, Szikora I, Kelly ME, Albuquerque FC, McDougall CG, et al: Curative cerebrovascular reconstruction with the Pipeline embolization device: the emergence of definitive endovascular therapy for intracranial aneurysms. J Neurointerv Surg 1:56-65, 2009

6. Guglielmi G, Viñuela F, Dion J, Duckwiler G: Electrothrombosis of saccular aneurysms via endovascular approach. Part 2: Preliminary clinical experience. J Neurosurg 75:8-14, 1991

7. Heller RS, Malek AM: Parent vessel size and curvature strongly influence risk of incomplete stent apposition in enterprise intracranial aneurysm stent coiling. AJNR Am J Neuroradiol 32:1714-1720, 2011

8. Jou LD, Mitchell BD, Shaltoni HM, Mawad ME: Effect of structural remodeling (retraction and recoil) of the pipeline embolization device on aneurysm occlusion rate. AJNR Am J Neuroradiol 35:1772-1778, 2014

9. Kühn AL, Hou SY, Puri AS, Silva CF, Gounis MJ, Wakhloo AK: Stent-assisted coil embolization of aneurysms with small parent vessels: safety and efficacy analysis. J Neurointerv Surg 8:581-585, 2016

10. Martínez-Galdámez M, Pérez S, Vega A, Ruiz P, Caniego 
JL, Bárcena E, et al: Endovascular treatment of intracranial aneurysms using the Pipeline Flex embolization device: a case series of 30 consecutive patients. J Neurointerv Surg 8:396-401, 2016

11. Papagiannaki C, Spelle L, Januel AC, Benaissa A, Gauvrit JY, Costalat V, et al: WEB intrasaccular flow disruptorprospective, multicenter experience in 83 patients with 85 aneurysms. AJNR Am J Neuroradiol 35:2106-2111, 2014

12. Sadato A, Hayakawa M, Adachi K, Kato Y, Hirose Y: Use of a new soft and long coil reduces the number of coils to embolize a small aneurysm. Interv Neuroradiol 21:161-166, 2015

13. Sheth SA, Patel NS, Ismail AF, Freeman D, Duckwiler G, Tateshima S: Treatment of wide-necked basilar tip aneurysm not amenable to Y-stenting using the PulseRider device. J Neurointerv Surg [epub ahead of print], 2015

14. Sivan-Hoffmann R, Gory B, Riva R, Labeyrie PE, Signorelli F, Eldesouky I, et al: One-year angiographic follow-up after WEB-SL endovascular treatment of wide-neck bifurcation intracranial aneurysms. AJNR Am J Neuroradiol 36:23202324,2015

15. Spiotta AM, Chaudry MI, Turk AS, Turner RD: Initial experience with the PulseRider for the treatment of bifurcation aneurysms: report of first three cases in the USA. J Neurointerv Surg 8:186-189, 2016

16. Yasumoto T, Osuga K, Yamamoto H, Ono Y, Masada M, Mikami K, et al: Long-term outcomes of coil packing for visceral aneurysms: correlation between packing density and incidence of coil compaction or recanalization. J Vasc Interv Radiol 24:1798-1807, 2013
17. Zhang J, Duan Y, Jin Z, Wei Y, Yang S, Luo J, et al: Stent boost subtract imaging for the assessment of optimal stent deployment in coronary ostial lesion intervention: comparison with intravascular ultrasound. Int Heart J 56:37-42, 2015

\section{Disclosures}

Dr. Aziz-Sultan is a proctor for Covidien/Medtronic and participates in the training of other physicians in the use of the liquid embolic agent Onyx as well as in the use of the Pipeline embolization device. No financial support was provided by Siemens.

\section{Author Contributions}

Conception and design: See, Aziz-Sultan. Acquisition of data: See, Dasenbrock, Ashour, Khandelwal, Patel, Frerichs, Aziz-Sultan. Analysis and interpretation of data: See, Silva, Dasenbrock, Khandelwal, Patel, Aziz-Sultan. Drafting the article: See, Silva. Critically revising the article: See, Silva, Dasenbrock, Ashour, Khandelwal, Patel, Aziz-Sultan. Reviewed submitted version of manuscript: all authors. Statistical analysis: See. Administrative/technical/material support: Aziz-Sultan. Study supervision: See, Frerichs, Aziz-Sultan.

\section{Correspondence}

Alfred P. See, Department of Neurosurgery, Brigham and Women's Hospital, Harvard Medical School, 75 Francis St., Boston, MA 02115. email: psee@partners.org. 